\title{
Developing a User Centric MultiSphere Model for Mobile Users Using a Visual Cell Platform
}

\author{
Seamus Hickey and Petri Pulli \\ Dept. of Information Processing Science, Uiversity of Oulu, FIN-90570 OULU, Finland
}

Key words: Mobile Services, Virtual reality, Multisphere model

\begin{abstract}
Research into $4^{\text {th }}$ generation mobile systems is expected to focus more attention on the development of user centred services as opposed to current network centric services Virtual reality technologies, like augmented reality and TeleReality, will play a greater role in the operation of these new services. By separating the hardware implementation platform from the service applications, it will be possible to deploy a wider range of services, broadening the scope and appeal of the system. This paper describes a service MultiSphere model for mobile users. The Service MultiSphere model uses virtual reality systems that are fundamentally based and coupled with the real world e.g. Augmented Reality and TeleReality. Three spheres of service are described, a Personal User Service Sphere, Local Environment Service Sphere and a Global Network Service. An experimental system based on Visual Cells is used to study the MultiSphere model
\end{abstract}

\section{INTRODUCTION}

As mobile communications industries and operators gear up for the introduction of $3^{\text {rd }}$ generation mobile systems, it is a relevant time for researchers to look towards $4^{\text {th }}$ generation systems. While researchers are concerned about many possible issues, there is a greater focus on the user, specifically the services that they will use and the interfaces necessary to use them. Future service provision will possibly place greater emphasis on bringing services from the network and surrounding environment closer to the mobile user $[1,2]$. It is envisioned that Virtual Reality technologies will 
play an increasingly important role in the shape and implementation of these services and the user interfaces that interact with them.

This paper describes a Service MultiSphere model for inherently real systems (Augmented [3] and TeleReality [4] systems). This concept is being proposed under the IST's Wireless Strategic Initiative (WSI) for $4^{\text {th }}$ generation mobile systems [1]. The Service MultiSphere model is 'user centric' in nature, i.e. the services revolve around the users personal and local environment. The MultiSphere model is broken down into 3 spheres divided between the user, the local environment and the global area network. The user is provided with a Personal User Service Sphere, which provides services only they can use without accessing any external network. The Local Environment Service Sphere provides the user with access to services that are available in the surrounding environment and the Global Service Network (GSN) provides access to global information and services like the Internet and UMTS.

A system to test and develop the MultiSphere service is under active construction as part of the PAULA project at the University of Oulu, Finland.

\section{USER CENTRIC DESIGN}

It would be good to reflect on what kinds of services are being offered by $2^{\text {nd }}$ and $3^{\text {rd }}$ generation mobile systems. Current $2^{\text {nd }}$ Generation systems (GSM) primarily provide user to user services like voice, messaging. $3^{\text {rd }}$ generation systems provide a richer multimedia support allowing access to the broader cyberworld space. Progressing on, $4^{\text {th }}$ generation should lead to more intuitive information services with a greater user centric dimension.

A user centric approach attempts to make the user the centre of the information/service world. Current mobile systems effectively place the emphasis on the user to access their network in order to use a service. The user centric approach forces the services to use the network to provide information to the user, at which point the user may decide what they will or will not avail of. Here is a subtle shift in emphasis away from the current approach of 'getting access' to the service, to 'bringing' the service to the user.

To explain further it would benefit us to examine how people acquire information in everyday life. People acquire enormous amounts of 
information from their surroundings by looking at various signs, media, advertisements, price tags etc. This is the basic first layer of information that the user is presented with. To acquire additional information, it is necessary to read package details, question sales representatives, check timetables etc., the second layer of information. Additionally the users themselves, may carry tools and devices that assist them in everyday activities, e.g. shopping lists, pens, mobile phones, PDA's, wallets, credit cards etc. In short the real environment that surrounds a person contains an incredible amount of information and service centred towards them.

\section{SERVICE MULTISPHERE MODEL}

The Service MultiSphere Model is based on a user centric approach and shown in Figure 1; is a variation of the MultiSphere model put forward in the WSI Book of Visions for $4^{\text {th }}$ generation Mobile systems [1]. The model shown in Figure 1, places the user in the centre of the sphere, highlighting their importance to the design approach. Surrounding the user is the Personal User Service sphere. The next layer is the Immediate Local Area Sphere, this represents the services available in the immediate location of the user (10-20 meters). The Local Area sphere contains services that are available from the general local area that the user is in, e.g. building's, street. It is larger in size than the immediate area, yet still covers a realistic geographical area surrounding the user. The final sphere, Global Service Network, covers services that are available globally, usually requiring access to a network such as UMTS, internet etc.

Personal User Service Sphere. The Personal User Sphere describes all of the services that on the mobile user. These are services that the mobile user essentially owns and are available from their own Personal Area Network (PAN) [5]. Only the user may access these services. By using Augmented Reality technology and combining the concept of a future generation media phone like Cyphone [2], the mobile user can divide their services into modes, e.g. a mobile user can carry the interface to their office around them. After work they can switch to an interface mode for shopping, entertainment, sport etc. 


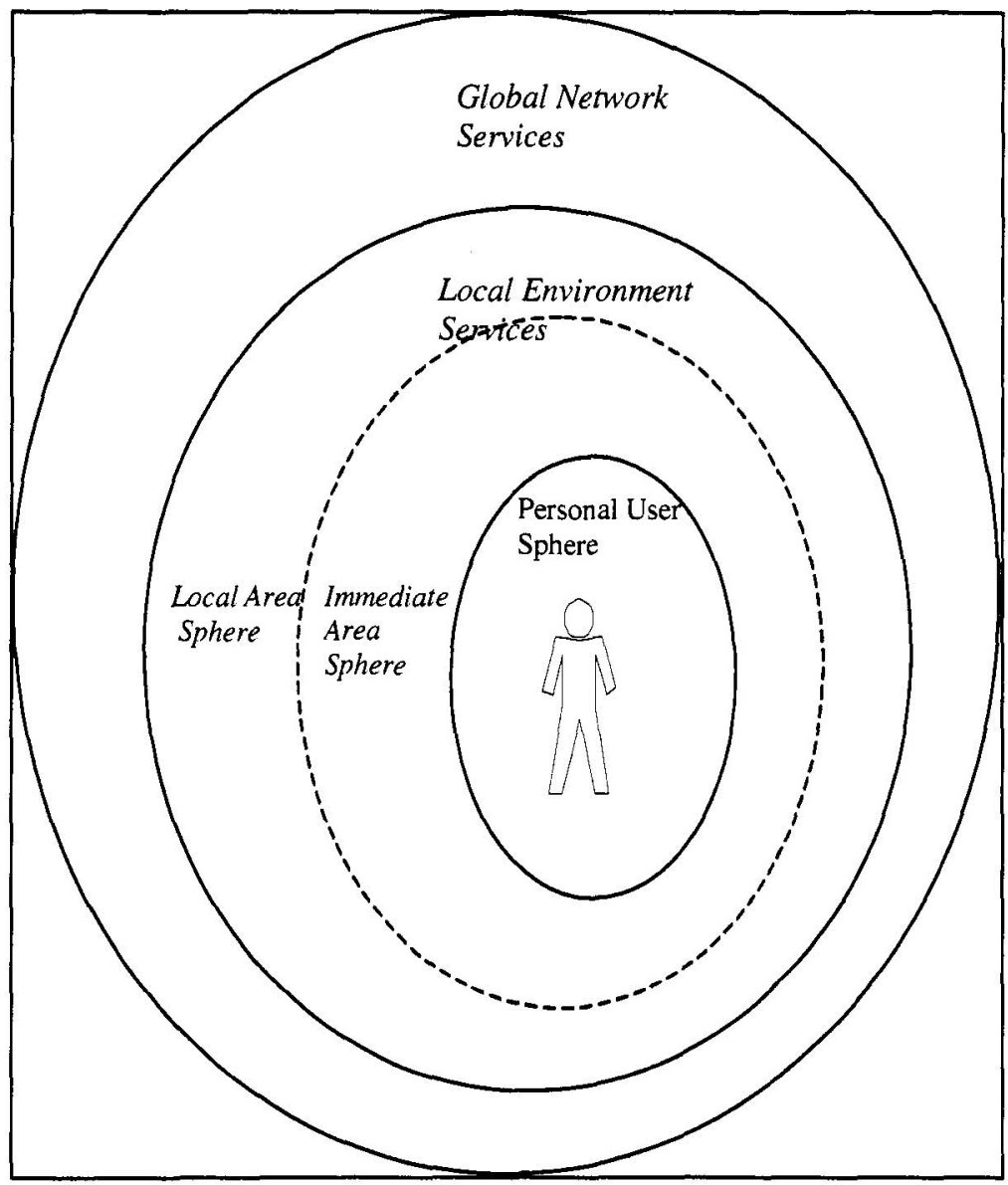

Figure 1. Service MultiSphere model for 4th generation mobile Systems

Local Environment Services. The environment area about the user can provide an immense amount of information. This environment contains 2 spheres, Immediate Area Service Sphere and Local Area Services Sphere. The user will only have access to these services if they 'visit' the location, at which point they may choose to make use of the provided service e.g. a public printer. The service is made known to the user yet it is up to the user to decide if they are willing to make use of that service and only afterwards would they be expected to pay any fee.

The Local Area Sphere covers a larger geographical area e.g. a building, street shop etc. The Local Area Sphere can provide general services and information which are available from that location as well as provide some 
assistance for location navigation, helping a user to find a shop, highlight which shops have sales, latest company news etc.

The Immediate Service Sphere is a small geographical area that contains some services specific to that particular spot e.g. supermarket grocery section. A Local area service and immediate area service can both coexist in the same area, yet offer different services

There is the potential for the user to receive an immense amount of information from the surrounding environment. It is important that the user can filter out its information so that they acquire relevant needed information. In essence the local area services are the providers of information, which the user must then sort out according to their needs.

Global Services Network. The Global Service Networks are services that are available from more universal systems like UMTS and the Internet.

The advantage of this service layer structure is that it divides services according to the area and needs of the user. With personal services, the user has total control to customise their settings to match their own needs. Area services provide additional user information e.g. company announcements, shop advertising etc. Global services can be accessed at anytime anywhere.

The service layers outlined are not without some problems and disadvantages. Two main concerns involve service information overload and security concerns. Service information overload can be caused by the user being swamped with information, which is either unwanted or is excessive enough that the user has difficulty in selecting the required service. Secondly, there is always the risk of a big brother feeling or that people may get access to services that they are not entitled to e.g. restricted areas. Hence some services could be placed on different levels of security access.

\section{EXPERIMENTAL SERVICE PLATFORM}

The PAULA project at the University of Oulu, Finland, is building an experimental system to examine the plausibility of a service MultiSphere. The service MultiSphere is partly implemented in the Visual cell project. 


\subsection{Visual cell}

The visual cell system is a TeleReality system [4]. The preferred method for implementing a TeleReality system at present is to arrange a large number of visual cells in a matrix to create a much larger space. A visual cell is defined as

'A limited space containing an image capturing device, where the space contains interactive real/virtual objects. Visual cells can be combined in a matrix to form one space that can be navigated and interacted with.'
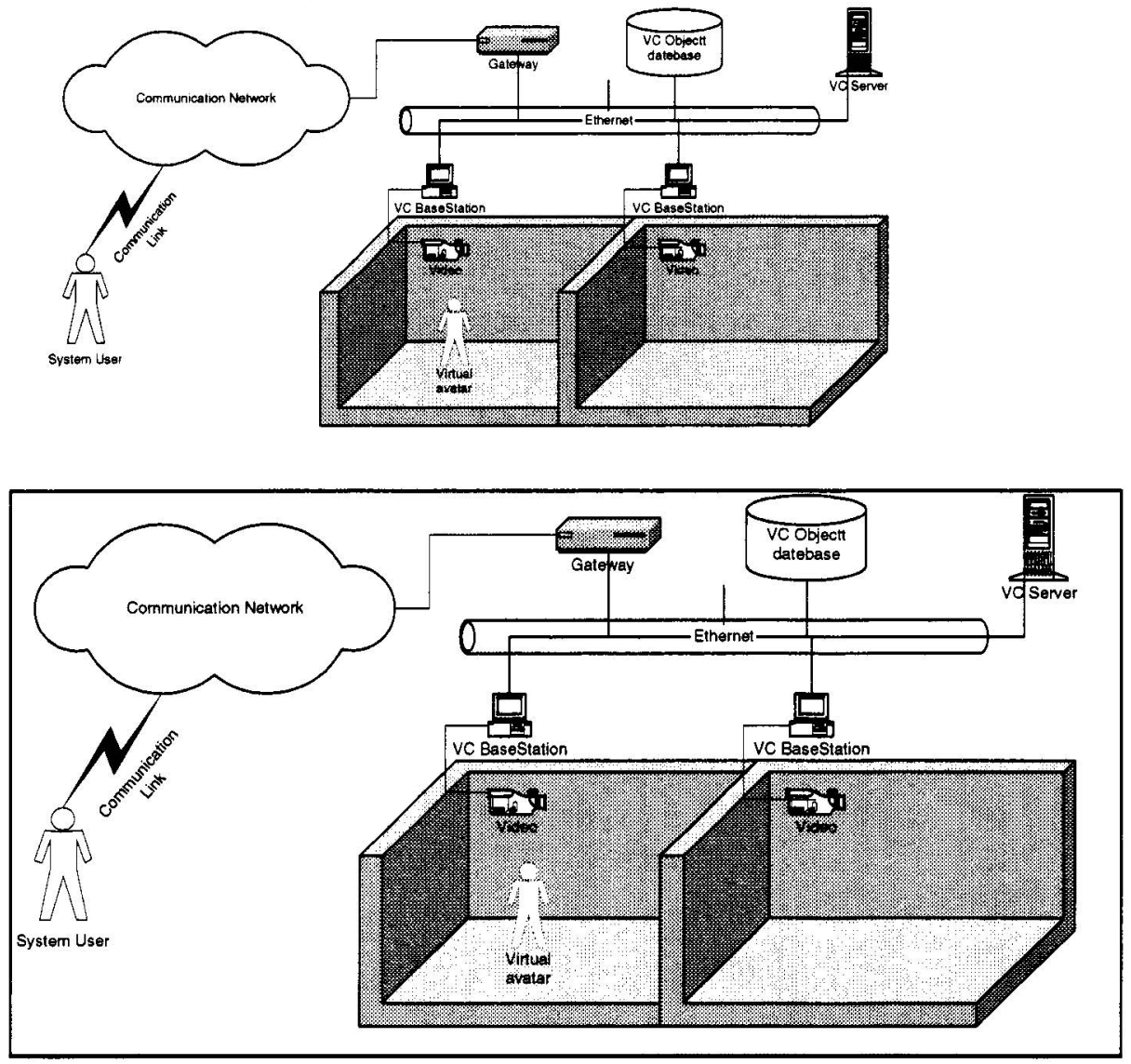

Figure 2. Experimental Visual cell architecture

An important element of this approach was to incorporate interactive objects that would allow a visitor to interact with the immediate surroundings. It soon became apparent however that when the user resided at the same location he was accessing, they were essentially receiving the same type of services as an Augmented Reality user. As an augmented reality 
system also uses a similar cell like structure to determine registration and location information, it was logical to group the service provision for both systems under the same service provision cell like structure.

The first version of an experimental Visual cell system containing 2 cells has been constructed. The system contains 2 visual cells, each providing a constant video feed. Each cell contains 1-2 interactive objects, used to 'teleport' the user from cell to cell. It is also possible to move from cell to cell, using keyboard keys. Figure 2 shows the current setup for the system.

Each visual cell is equivalent to an Immediate Area Sphere while the Local Area Sphere resides in the VC Server. Access to the GSN is available by TCP/IP, although no services use this feature yet. The database stores the visual cell object information, which is accessed at start up. In future iterations of the system, it is planned to store dynamic objects and migrating objects in a 'visiting' database

The Visual cell system provides a good starting point for further research and conceptual testing

\section{CONCLUSIONS}

This paper has set out to present a service MultiSphere structure for use with inherently real technologies like Augmented Reality and TeleReality. The service layer composes of 3 different core layers, the personal service layer, Area layer and global Layer. The Area layer is decomposed into cells, which provide services at specified locations while the over layer provides universal services for the whole area. In addition, an experimental platform system using visual cells was introduced. The visual cell concept was described and the architecture of the system was discussed. Considerable work is still required to fully develop the MultiSphere concept and visual cell system. For the visual cell system, future work will primarily focus on the details of service components as well as the addition of short-wave radio to allow an augmented reality user access to the system.

\section{ACKNOWLEDGEMENTS}

This project is sponsored by the PAULA project. The PAULA project is supported by the Academy of Finland and is a co-operative work between 
the University of Oulu, Helsinki University of Technology, Tamper University of Technology

\section{REFERENCES}

[1] IST WSI 2000, 'Book of Visions 2000', http://www.ist-wsi.orp/

[2] Pulli P., Pyssysalo T.,Similä J.,Metsavainio J. 1998, 'CyPhone - Future Personal Telecooperation Device', Telecooperation. Proceedings of the XV.IFIP World Computer Congress, Vienna \& Budapest

[3] Azuma R. T. 1997. 'A Survey of Augmented Reality'. Presence: Teleoperators and Virtual Environments. Vol 6, No. 4, August 1997, pg. 355-385. MIT Press

[4] Hickey S, Manninen T, Pulli P. 2000. 'TeleReality - The next step for Telepresence'. Proceedings of the World Multiconference on Systemics, Cybernetics \& Informatics (VOL 3) (SCI 2000), pp 65-70, Florida.

[5] Banatre M., Couderc P., Weis F., 2000. 'Spontaneous Communications', IST Mobile Communications Summit 2000, pp.789-793, Galway Ireland 